\title{
Editorial: an obsession with subtyping gastric cancer
}

\author{
Haruhiko Sugimura
}

Published online: 13 March 2013

(c) The International Gastric Cancer Association and The Japanese Gastric Cancer Association 2013

Keywords Subtype - Histopathology · Microsatellite instability - Chromosomal numerical abnormality . General rules

My English revisers always correct my ambiguous usage of "type" and "subtype" to describe cancers. I sometimes use "cancer type" in the context of adenocarcinoma versus squamous cell carcinoma, but at other times I use it in the context of gastric cancer versus lung cancer. I am not sure whether to refer to "adenocarcinoma of the lung" as a type or a subtype when discussing a grandiose topic such as the "histopathology of human cancer."

Categorization was part of human nature even before the work of Carl von Linné, but no categorizations can be more complicated than pathological categorizations, especially those based on the microscopic morphology of cancer cells. Why do we categorize tumors? Lung cancer and gastric cancer are different, so their therapy, care, and prevention measures should also differ. Why must we differentiate between adenocarcinoma and squamous cell carcinoma among cancers arising within the same organ (such as the lung)? Again, therapy (probably), care (possibly), and prevention measures (definitely: for example, avoiding smoking to prevent squamous cell carcinoma of the lung) should be modified according to the tumor category. Then, should we also discriminate among the subtypes of adenocarcinoma of the gastrointestinal tract, such as well-

This editorial refers to the article doi:10.1007/s10120-012-0226-6.

\section{H. Sugimura $(\bowtie)$}

Department of Tumor Pathology, Hamamatsu University

School of Medicine, 1-20-1 Handayama, Higashi-ku,

Hamamatsu 431-3192, Japan

e-mail: hsugimur@hama-med.ac.jp differentiated versus poorly differentiated? Yes, we know of biological differences between these two subtypes, and a principle of general pathology teaches us that the latter is generally associated with a poorer prognosis. However, we are not sure whether this difference is notable when comparing subtypes at different stages (for example, earlystage poorly differentiated cancer versus advanced-stage well-differentiated cancer). Heterogeneity within a single tumor and heterogeneity among many similar-looking tumors had been known before the era of massive parallel sequencing revolutionized molecular concepts regarding human cancer.

One of my good friends, a confident pulmonary surgeon, once said to me, "No histological description is needed in a breast cancer pathology report; just 3 or 4 immunohistological scores including HER2, hormone receptors, and maybe a proliferation indicator, such as the Ki-67 labeling index." In the era of companion diagnostics, categorization based on morphology, which is often subjective, may only further complicate clinical cancer management and create an unnecessary burden on pathologists. Actually, every few years the name, subtype categories, and requirements for describing questionable attributes change for unknown reasons and without any emerging evidence but simply because they have been labeled as being a "general rule." On the other hand, as we approach the brink of personal medicine, the ultimate tumor subtyping-in which the whole genome sequencing of tumor DNA up to the singlecell level will be performed-is appearing on the horizon. The cost of such analyses is becoming less and less and is much less than that of hiring technicians who can make beautifully stained sections. In contrast to morphological classifications, the DNA sequence data can directly pinpoint target molecules that clinicians can then use as a starting point for individualized therapy. Complaints of late 
or insufficient pathology reports will disappear, along with the jobs of pathologists. How, then, should the current morphological subtyping be viewed from a molecular perspective?

The first edition of the international histological classification of tumors of the upper gastrointestinal tract, published in 1977, was led and edited by a Japanese pathologist, Dr. Kunio Oota, and pathologists in 13 countries [1]. Dr. Isamu Kino, who was one of the consulting pathologists of that issue, collected and viewed the glass slides of the gastric tumors and then discussed the diagnosis or subtyping with the other participants. The subtypes described for adenocarcinoma continue to be used today [2]: (a) papillary, (b) tubular, (c) mucinous, and (d) signetring cell carcinoma. However, the TNM classification prefers a simpler grading: well, moderately, and poorly differentiated [3]. The simplicity of a classification is very important for clinical practice, and the general view of tumors differs according to the stages that are typically encountered by pathologists, which in turn is influenced by the health insurance system, the surveillance system, and the proficiency of diagnostic and screening clinicians in each country. Thus, histological subtypes that can withstand the austerity of medical cost measures must have both a biological and a therapeutic relevance.

In this issue, Tomio Arai, one of the greatest pupils of the late Prof. Kino, has described biological characteristics that are closely related to the morphological subtype. Arai describes a high prevalence of microsatellite instability (MSI) in the papillary subtype of well-differentiated adenocarcinoma and in the solid subtype of poorly differentiated adenocarcinoma of the stomach [4]. Dr. Arai and his colleagues took advantage of a particular series of surgical cases at a single institute, which mainly treats the elderly, and they clearly characterized MSI-positive tumors, which tended to occur in older patients and exhibited a female preponderance and particular histological features. As previously reported, MSI in earlystage adenocarcinoma with papillary features (papillary subtype of well-differentiated adenocarcinoma) is often caused by MLH1 promoter methylation [5], and the concept of field cancerization arising from epigenetic changes has now been extended to and established for other cancers and genes [6]. Arai further showed that a solid subtype of poorly differentiated adenocarcinoma that usually appears as a component in a progressive stage also gains MSI during tumor growth. This finding in solid-subtype tumors implies that the solid subtype, which is usually assigned to a diffuse-type category, should be reassigned as an intestinal type based on the features of its molecular lineage.

Their clear demonstration and meticulous pathological analysis have brought about an end to arguments regarding
MSI and histological features. Earlier reports often concluded that MSI was more prevalent in poorly differentiated, diffuse-type tumors than in intestinal-type tumors [7]. This dichotomy of gastric cancer classification, intestinal versus diffuse type, has often been used by the international community, and the morphological features of the "subtypes" of both categories are often missed. Furthermore, in advanced gastric cancer, the tissues often have a heterogeneous morphology (Prof. Kino often referred to this as a "varied structure"), and they are often MSI positive, which might reflect clonal heterogeneity because the extra bands on the gel are interpreted as evidence of CA repeat slippages [8]. The detailed description of the tissue, including an exact measurement of MSI, is often inadequate, as many laboratory investigators have experienced.

Another issue that Arai addressed is the relationship between MSI and the patient prognosis. Prejudice based on early reports of MSI-positive colon cancer might lead us to expect that MSI-positive tumors are usually associated with a biologically better prognosis [9]. The findings of a better prognosis in MSI-positive cancer cases are probably related to the fact that these MSI-positive cancer cells harbored a near-diploid pattern of DNA. Instead of chromosomal numerical abnormality (CNA), which is related to the extraordinary destruction of genetic material and is strongly correlated with an abysmal prognosis [10], authentic MSI-positive tumors often exhibit a less drastic copy number change in chromosomes [11]. The stageadjusted analysis performed by Arai et al. did not support a better prognosis for MSI-positive cases in their study. MSIpositive, papillary (sub)type gastric cancer exhibited fewer CNA; thus, CNA information for MSI-positive tumors would be interesting.

The data Arai and his colleagues have provided here will help pathologists to understand the relevance of the subtyping of well-differentiated gastric adenocarcinoma, and this kind of basic support for attributes that many young clinical doctors wrongly believe to be scientifically sound remains scarce in the "general rules" of clinical practice, as most of the required attributes are mainly for research purposes. We should continue to make an effort to validate the significance or insignificance of these attributes so as to edit out the unnecessary ones. Even scientifically sound data, such as those reported by Arai et al., do not necessarily need to be included in conventional pathology reports. Only when these markers are recognized as determinants for the selection of therapeutic measures by several follow-up studies should such obsession with classification become a rule.

Finally, the high autopsy rate at the institution of Arai et al. has provided us with the opportunity to see the natural history and final consequences of cancer in Japanese patients. We congratulate their achievements. 
Acknowledgments This contribution was supported, in part, by the Grants-in-Aid for the U.S.-Japan Cooperative Medical Science Program; the National Cancer Center Research and Development Fund; Grant for Priority Areas from the Japanese Ministry of Education, Culture, Sports, Science and Technology (221S0001); and Grants-inAid for Cancer Research from the Japanese Ministry of Health, Labour, and Welfare (23120201 and 10103838), and the Princess Takamatsu Cancer Research Fund.

\section{References}

1. Oota K, Sobin LH. Histological typing of gastric and oesophageal tumors. Geneva: World Health Organization; 1977.

2. Bosman FT, Carneiro F, Hruban RH, Theise ND. WHO classification of tumours of the digestive system. Lyon: IARC; 2010.

3. TNM classification of malignant tumors. 6th edn. New York: Wiley; 2002.

4. Arai T, Sakurai U, Sawabe M, Honma N, Aida J, Ushio Y, et al. Frequent microsatellite instability in papillary and solid-type, poorly-differentiated adenocarcinomas of the stomach. Gastric Cancer. 2013. doi:10.1007/s10120-012-0226-6.

5. Guo RJ, Arai H, Kitayama Y, Igarashi H, Hemmi H, Arai T, et al. Microsatellite instability of papillary subtype of human gastric adenocarcinoma and hMLH1 promoter hypermethylation in the surrounding mucosa. Pathol Int. 2001;51(4):240-7.
6. Ushijima T, Hattori N. Molecular pathways: involvement of Helicobacter pylori-triggered inflammation in the formation of an epigenetic field defect, and its usefulness as cancer risk and exposure markers. Clin Cancer Res. 2012;18(4):923-9.

7. Han HJ, Yanagisawa A, Kato Y, Park JG, Nakamura Y. Genetic instability in pancreatic cancer and poorly differentiated type of gastric cancer. Cancer Res. 1993;53(21):5087-9.

8. Wang Y, Shinmura K, Guo RJ, Isogaki J, Wang DY, Kino I, et al. Mutational analyses of multiple target genes in histologically heterogeneous gastric cancer with microsatellite instability. Jpn J Cancer Res. 1998;89(12):1284-91.

9. Boland CR, Thibodeau SN, Hamilton SR, Sidransky D, Eshleman JR, Burt RW, et al. A National Cancer Institute Workshop on Microsatellite Instability for cancer detection and familial predisposition: development of international criteria for the determination of microsatellite instability in colorectal cancer. Cancer Res. 1998;58(22):5248-57.

10. Suzuki M, Nagura K, Igarashi H, Tao H, Midorikawa Y, Kitayama $\mathrm{Y}$, et al. Copy number estimation algorithms and fluorescence in situ hybridization to describe copy number alterations in human tumors. Pathol Int. 2009;59(4):218-28.

11. Song JP, Kitayama Y, Igarashi H, Guo RJ, Wang YJ, Kobayashi $\mathrm{T}$, et al. Centromere numerical abnormality in the papillary, papillotubular type of early gastric cancer, a further characterization of a subset of gastric cancer. Int J Oncol. 2002;21(6):1205-11. 